\title{
ORIGINALES
}

\section{Recursos de osteosíntesis en fémur porótico}

\author{
P. Tornera, X. Gallarta, S. Sastre ${ }^{a}$, S. García ${ }^{a, b}$, J.M. Segurb, J. Riba y S. Suso ${ }^{a, b}$ \\ anidad de Cadera $y^{b}$ Banco de Tejidos del Aparato Locomotor. \\ Servicio de Cirugía Ortopédica y Traumatología. Instituto Clínic del Aparato Locomotor \\ Hospital Clínic. Universidad de Barcelona.
}

Objetivos. Exponer dos técnicas quirúrgicas de refuerzo de una osteosíntesis convencional en situaciones especiales de debilidad ósea del fémur, como son las fracturas periprotésicas, las fracturas patológicas y los fracasos de osteosíntesis previas en pacientes de edad avanzada. La debilidad del hueso puede llegar a dificultar la realización de osteosíntesis estables en fracturas de fémur porque impide el correcto anclaje del material, ya sean agujas, cerclajes o especialmente tornillos.

Material y método. Se evaluaron 13 pacientes (media de edad de 78,3 años), 9 de los cuales sufrieron fracturas periprotésicas de fémur, dos fracasos de osteosíntesis previas, un caso de pseudoartrosis tras una fractura periprotésica y una fractura diafisaria en un fémur muy osteoporótico, en los cuales se han utilizado dos técnicas de refuerzo del hueso: el cementado endomedular y el implante de contraplacas atornilladas de aloinjerto óseo congelado.

Resultados. Se realizó un seguimiento de estos pacientes en un período que oscila entre 12 y 72 meses (media de 26,2 meses). En un caso se produjo una pseudoartrosis de la fractura por ocupación accidental del foco de fractura por el cemento acrílico. En los 12 casos restantes se consiguió la consolidación.

Conclusiones. El cementado endomedular y la utilización de contraplacas atornilladas de aloinjerto óseo son dos recursos técnicos útiles en situaciones especiales en las que es necesario realizar una osteosíntesis estable sobre un hueso muy débil.

Palabras clave: fractura, periprotésica, fémur, aloinjerto óseo, osteoporosis.

\section{Osteosynthesis resources for osteoporotic femur}

Objectives. To describe two surgical techniques for reinforcing conventional bone fixation in special situations of femoral bone weakness, such as periprosthetic fracture, pathologic fracture, and failure of previous bone fixation in patients of advanced age. Bone weakness can make it difficult to achieve stable osteosynthesis of femoral fractures because it impedes correct fixation of materials, whether pins, tension bands or, especially, screws.

Materials and methods. Thirteen patients (mean age 78.3 years) were evaluated, 9 with periprosthetic fracture of the femur, 2 osteosynthesis failures, 1 nonunion after periprosthetic fracture, and 1 diaphyseal fracture in an osteoporotic femur. Two techniques were used to reinforce the bone: intramedullary cementing and the implantation of screw-on counterplates of frozen bone allograft.

Results. Patients were followed up for 12 to 72 months (mean 26.2 months). In one patient, nonunion occurred due to accidental occupation of the fracture site by acrylic cement. The remaining 12 patients achieved bone healing.

Conclusions. Intramedullary cementing and the use of screw-on counterplates of bone allograft are two useful technical resources for special situations requiring stable bone fixation of very weak bone.

Key words: fracture, periprosthetic, femur, bone allograft, osteoporosis.

\footnotetext{
Correspondencia:

P. Torner.

Servicio de Cirugía Ortopédica y Traumatología.

Instituto Clínic del Aparato Locomotor. Hospital Clínic.

C/ Villarroel, 170

08036 Barcelona.

Correo electrónico: 29817ptp@ comb.es
}

Recibido: junio de 2003.

Aceptado: octubre de 2003.
Las condiciones particulares de determinados pacientes (edad muy avanzada, osteoporosis, deterioro general, metástasis, etc.) pueden dificultar en gran medida la osteosíntesis de una fractura de fémur a causa de la debilidad del hueso (tabla 1). Ésta puede convertir una osteosíntesis correcta en precaria e inestable y llevarla al fracaso si el método de fijación empleado no tiene un buen anclaje en el hueso. Además, no existe consenso sobre el tratamiento óptimo para este tipo de fracturas ${ }^{1-9}$. 
Tabla 1. Factores causales de debilidad ósea

Cirugía de revisión

Artritis reumatoide

Tratamiento corticoideo

Aumento de la fragilidad ósea (osteoporosis, osteomalacia, etc.)

Osteolisis localizada

Perforación de la cortical

Aumento del estrés cortical (sobrecarga del extremo de la prótesis por alteración del centraje, extremos de las placas, osteolisis de tornillos)

Deformidades del fémur proximal (fracturas previas, osteotomías, displasias)

Descementación de la prótesis (enfermedad de las partículas)

Hemos aplicado en 13 casos de fracturas sobre hueso debilitado dos recursos técnicos con el objetivo de conseguir un anclaje firme de los tornillos. El primero es el cementado endomedular (con cemento de polimetilmetacrilato) de los dos fragmentos principales de la fractura, consiguiéndose un buen anclaje de los tornillos, lo que permite realizar una osteosíntesis estable con placa siguiendo los principios básicos habituales. En el caso de las fracturas periprotésicas la cavidad medular ya está ocupada, y por tanto su cementado no es posible. En estos casos aplicamos una contraplaca larga de hueso cortical homólogo congelado adaptada a la cara medial del fémur y que permite conseguir también un anclaje óptimo de los tornillos. El objetivo del trabajo es exponer nuestra experiencia con estos dos métodos de refuerzo óseo que permiten conseguir osteosíntesis estables incluso en casos especiales de extrema debilidad del hueso.

\section{MATERIAL Y MÉTODO}

Entre enero 1996 y diciembre de 2001 hemos utilizado dichos recursos técnicos en 13 ocasiones. La técnica del cementado endomedular (fig. 1) se ha realizado en 5 pacientes (tabla 3, casos 1 al 5). Se trata de 5 mujeres, con una edad media de 86 años: dos fracturas periprotésicas distales a una prótesis de cadera (tipo $\mathrm{C}$, según la clasificación de Vancouver ${ }^{10}$ ) (tabla 2), dos fracasos de osteosíntesis (un clavo

Tabla 2. Clasificación de Vancouver de fracturas periprotésicas de fémur proximal

Tipo A: afectación región trocantérea

AG: afectación del trocánter mayor

AL: afectación del trocánter menor

Tipo B: fracturas alrededor del vástago o ligeramente distales

B1: implante no aflojado

B2: implante aflojado, buen stock óseo

B3: implante aflojado, mal stock óseo

Tipo C: fracturas distales a la punta del vástago, pudiéndose ignorar el mismo para el tratamiento

Tomada de Duncan CP, Masri BA ${ }^{10}$.

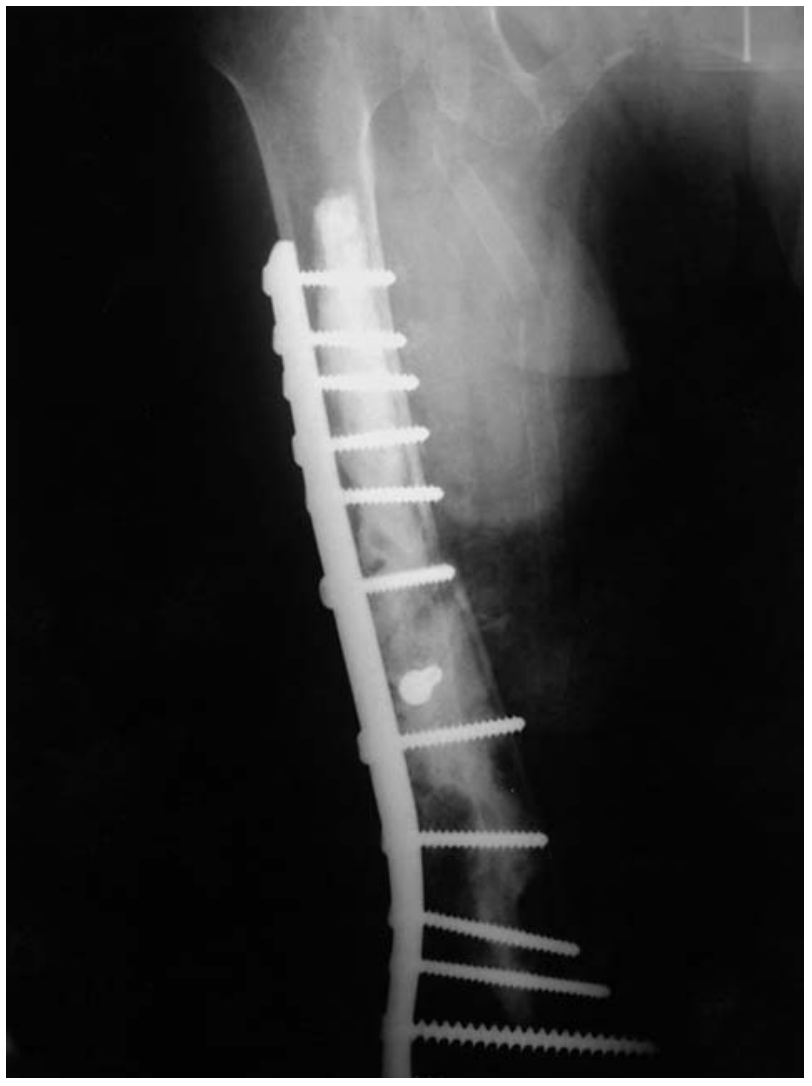

Figura 1. Fractura diafisaria espiroidea de fémur en paciente de 88 años (tabla 3, caso n. ${ }^{\circ} 1$ ). Osteosíntesis con cementado endomedular y placa. Consolidación a los 4 meses, sin complicaciones.

endomedular en fractura subtrocantérea y una síntesis con placa en una fractura diafisaria) y un caso de una fractura diafisaria de fémur en hueso muy porótico que no permitía el correcto anclaje de los tornillos.

Hemos aplicado la técnica de osteosíntesis con placa y contraplaca de aloinjerto (fig. 2) en 8 pacientes (tabla 3, casos 6 al 13) (3 varones y 5 mujeres, con una edad media de 72,7 años). Todos eran casos de fracturas periprotésicas de fémur (5 casos tipo B1, 1 caso tipo B2 y 2 casos tipo B3, según la clasificación de Vancouver $\left.^{10}\right)$. Describimos a continuación las dos técnicas quirúrgicas.

\section{Cementado endomedular}

La identificación exacta del tipo de fractura y la planificación preoperatoria de la osteosíntesis a realizar son de máxima importancia. Se limpia cuidadosamente el foco de fractura, se legra la cavidad medular con cuchara para obtener una limpieza previa al cementado y a continuación se procede a la reducción de la fractura para asegurar que ésta es posible sin dificultad. Se prepara cemento acrílico y se inyecta desde el foco de fractura a los dos fragmentos principales, en sentido proximal y distal. Se reduce la fractura y se mantiene durante el fraguado del cemento, tenien- 
do especial cuidado en evitar que el cemento entre en contacto con el foco de la fractura para permitir la consolidación. Posteriormente se moldea la placa de osteosíntesis y se procede a su colocación según la técnica habitual, con compresión interfragmentaria y axial de la fractura (añadiendo injerto autólogo en fracturas transversales y oblicuas cortas). La perforación del cemento y su posterior terrajado permiten un anclaje óptimo de los tornillos. La pauta postoperatoria de sedestación, inicio de la marcha, carga sobre la extremidad, etc., se realiza como si se tratase de una osteosíntesis realizada sobre hueso sano.

\section{Contraplaca atornillada de hueso homólogo congelado}

De nuevo es imprescindible una cuidadosa planificación preoperatoria. Se identifica y limpia el foco de fractura, se reduce y se mantiene la reducción mediante pinzas. Se diseca la cara anterior y medial del fémur liberándola de las inserciones musculares del cuádriceps. A continuación se talla una placa larga de diáfisis de hueso homólogo de al menos $3 \mathrm{~cm}$ de ancho. Preferimos utilizar aloinjertos de diáfisis tibial porque es más fácil conseguir una forma plana que se adapte fá- cilmente al fémur del paciente. Se introduce la placa de aloinjerto deslizándola por la cara anterior del fémur hasta situarla en su posición definitiva en la cara medial. A continuación se coloca la placa de osteosíntesis en la cara externa del fémur. Esta disposición se mantiene mediante pinzas de reducción o cerclajes alámbricos (que opcionalmente podrán ser retirados después). Se introducen inicialmente los tornillos de compresión, interfragmentaria y axial. Estos tornillos deben fijarse únicamente al hueso del paciente, puesto que su anclaje en la contraplaca de aloinjerto impediría la compresión de la fractura. A continuación, con la fractura ya estabilizada y comprimida, se procede a colocar el resto de tornillos para la neutralización, que deben anclarse en la contraplaca de aloinjerto. El anclaje es muy firme y esto permite realizar una osteosíntesis estable. Como en el caso anterior, se aplica el protocolo postoperatorio habitual como si se tratase de una osteosíntesis realizada sobre hueso sano.

\section{RESULTADOS}

Hemos realizado osteosíntesis con cementado endomedular del fémur en 5 pacientes, con un seguimiento medio

Tabla 3. Pacientes incluidos en este estudio

\begin{tabular}{|c|c|c|c|c|c|c|c|}
\hline & Edad & Sexo & Diagnóstico & Comentarios & $\begin{array}{l}\text { Tipo de } \\
\text { fractura }\end{array}$ & & Seguimiento \\
\hline 1 & 88 & M & Fractura diafisaria fémur & Osteoporosis grave & $32-\mathrm{B} 1.2 *$ & $\begin{array}{l}\text { Placa osteosíntesis + cementado } \\
\text { endomedular }\end{array}$ & 26 meses \\
\hline 2 & 85 & M & $\begin{array}{l}\text { Fallo osteosíntesis (clavo } \\
\text { endomedular) }\end{array}$ & $\begin{array}{l}\text { Fallo encerrojado distal } \\
\text { inicial }\end{array}$ & $32-\mathrm{B} 1.1 *$ & $\begin{array}{l}\text { Placa osteosíntesis + cementado } \\
\text { endomedular }\end{array}$ & 27 meses \\
\hline 3 & 81 & M & $\begin{array}{l}\text { Fallo osteosíntesis (placa } \\
\text { osteosíntesis) }\end{array}$ & Osteoporosis grave & $32-\mathrm{A} 3.2 *$ & $\begin{array}{l}\text { Placa osteosíntesis + cementado } \\
\text { endomedular }\end{array}$ & 24 meses \\
\hline 4 & 85 & M & Fractura periprotésica & PTC hace 6 años & $\mathrm{C}^{* *}$ & $\begin{array}{l}\text { Placa osteosíntesis + cementado } \\
\text { endomedular }\end{array}$ & 39 meses \\
\hline 5 & 91 & M & Fractura periprotésica & $\begin{array}{l}\text { Hemiartroplastia hace } \\
\quad 3 \text { años }\end{array}$ & $\mathrm{C}^{* *}$ & DCS $95^{\circ}+$ cementado endomedular & 14 meses \\
\hline 6 & 58 & V & Fractura periprotésica & PTC hace 6 años & $\mathrm{B} 1 * *$ & $\begin{array}{l}\text { Placa Dall-Miles + contraplaca } \\
\text { aloinjerto }\end{array}$ & 20 meses \\
\hline 7 & 81 & M & Fractura periprotésica & PTC hace 11 años & $\mathrm{B} 1 * *$ & $\begin{array}{l}\text { Placa osteosíntesis + contraplaca } \\
\text { aloinjerto óseo }\end{array}$ & 24 meses \\
\hline 8 & 61 & V & $\begin{array}{l}\text { Pseudoartrosis en fractura } \\
\text { periprotésica (cemento } \\
\text { endomedular en } 1 .^{\mathrm{a}} \\
\text { intervención) }\end{array}$ & $\begin{array}{l}\text { Fractura periprotésica } \\
\text { hace } 14 \text { meses }\end{array}$ & $\mathrm{B} 1 * *$ & $\begin{array}{l}\text { Placa osteosíntesis + contraplaca } \\
\text { aloinjerto óseo }\end{array}$ & 15 meses \\
\hline 9 & 66 & M & Fractura periprotésica & $\begin{array}{l}\text { Aflojamiento vástago } \\
\text { (PTC } 12 \text { años) }\end{array}$ & $\mathrm{B} 3 * *$ & $\begin{array}{l}\text { Recambio vástago + placa } \\
\text { osteosíntesis + contraplaca } \\
\text { aloinjerto }\end{array}$ & 12 meses \\
\hline 10 & 87 & M & Fractura periprotésica & $\begin{array}{l}\text { Hemiartroplastia hace } \\
2 \text { años }\end{array}$ & $\mathrm{B} 1 * *$ & $\begin{array}{l}\text { Placa osteosíntesis + contraplaca } \\
\text { aloinjerto }\end{array}$ & 23 meses \\
\hline 11 & 78 & M & Fractura periprotésica & PTC hace 10 años & $\mathrm{B} 1 * *$ & $\begin{array}{l}\text { Placa Dall-Miles + contraplaca } \\
\text { aloinjerto }\end{array}$ & 16 meses \\
\hline 12 & 81 & $\mathrm{~V}$ & Fractura periprotésica & $\begin{array}{l}\text { Aflojamiento vástago } \\
\text { (PTC } 10 \text { años) }\end{array}$ & $\mathrm{B} 3 * *$ & $\begin{array}{l}\text { Recambio vástago + placa } \\
\text { osteosíntesis + contraplaca } \\
\text { aloinjerto }\end{array}$ & 29 meses \\
\hline 13 & 70 & M & Fractura periprotésica & PTC hace 8 años & $\mathrm{B} 2 * *$ & $\begin{array}{l}\text { Placa osteosíntesis + contraplaca } \\
\text { aloinjerto }\end{array}$ & 72 meses \\
\hline
\end{tabular}

*Según clasificación AO. ** Según clasificación de Vancouver de fracturas periprotésicas de fémur proximal. PTC = prótesis total de cadera; V = varón; M = mujer. 


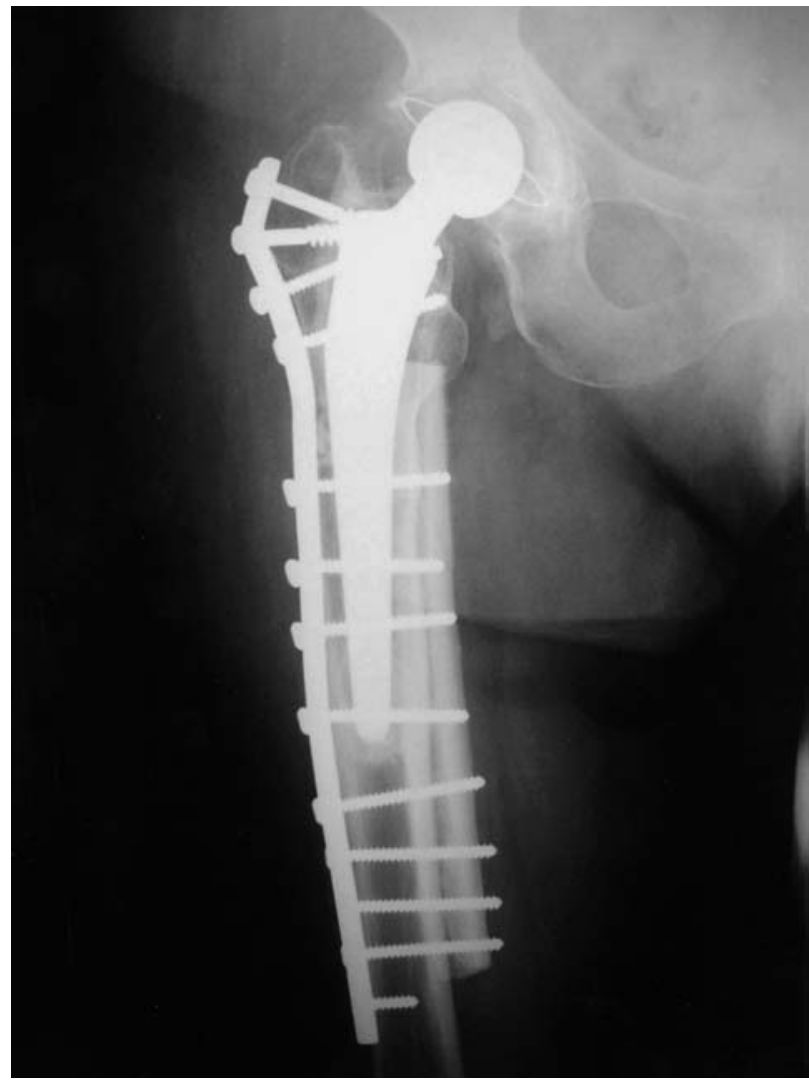

Figura 2. Fractura periprotésica de fémur (tipo B1) en paciente de 81 años (tabla 3, caso n. ${ }^{\circ}$ 7). Osteosíntesis con placa y contraplaca atornillada de aloinjerto óseo congelado.

de 26 meses (mínimo de 14 meses y máximo de 39 meses). En un caso de fractura subtrocantérea (tabla 3 , caso n. ${ }^{\circ}$ ) se produjo una varización de la fractura (con ángulo cervicodiafisario final de $115^{\circ}$ ), pero consolidó y el resultado final fue satisfactorio. Otro caso (tabla 3 , caso n. ${ }^{\circ}$ 8) evolucionó a pseudoartrosis y se produjo la rotura del implante 7 meses después de la intervención (figs. 3 y 4). Esto fue debido a la penetración accidental de cemento en el foco de la fractura durante la osteosíntesis, lo que impidió la consolidación. Solucionamos este caso comprimiendo la fractura, aportando autoinjerto de cresta ilíaca y realizando una nueva osteosíntesis con placa y contraplaca atornillada de aloinjerto óseo congelado (fig. 5). En el resto de pacientes se consiguió la consolidación de la fractura sin complicaciones (tiempo medio de consolidación de 4,5 meses).

La colocación de una contraplaca de aloinjerto en la cara medial del fémur nos ha permitido realizar osteosíntesis estables en casos complejos. Hemos aplicado esta técnica en 8 pacientes, con un seguimiento medio de 26,4 meses (mínimo de 12 meses y máximo de 72 meses), consiguiendo en todos ellos la consolidación de la fractura (tiempo medio de consolidación de 4,2 meses) y sin complicaciones. En los casos de seguimiento más prolongado, hemos podido obser-

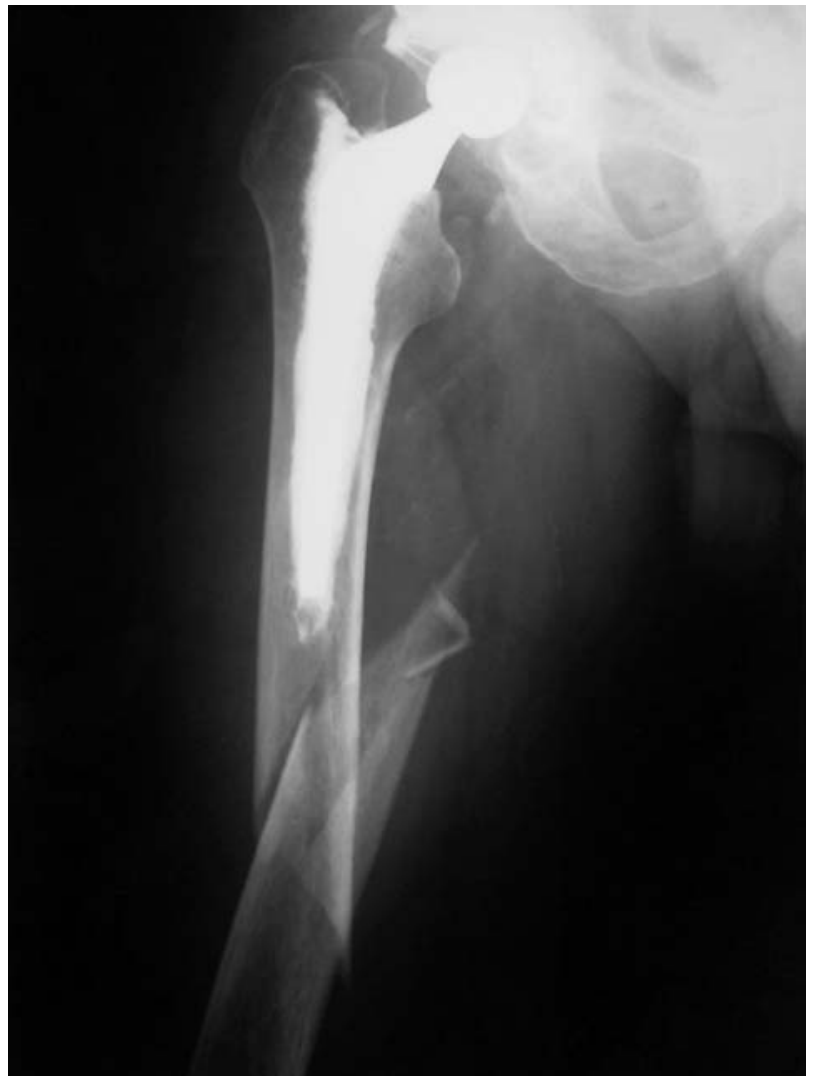

Figura 3. Fractura periprotésica en paciente de 61 años (tabla 3, caso $\left.n .{ }^{\circ} 8\right)$ sin signos de aflojamiento del vástago protésico. Se trató inicialmente mediante osteosíntesis con placa (ver figs. 4 y 5).

var la incorporación del aloinjerto, que se convierte así en refuerzo permanente del fémur del paciente (fig. 6).

\section{DISCUSIÓN}

La osteosíntesis de fracturas en hueso porótico plantea con frecuencia dificultades de tratamiento. Actualmente en muchos de estos casos se recurre a una osteosíntesis mediante enclavado endomedular, cuyo éxito depende menos de la calidad del hueso en el que asienta, ya que proporciona una estabilidad relativa aun sin compresión interfragmentaria ${ }^{11}$. Sin embargo, en otros pacientes (fracturas periprotésicas, complicaciones de osteosíntesis previas) este tipo de osteosíntesis no es posible y no existe consenso sobre el tratamiento a utilizar ${ }^{1-9}$. Además, cualquier fractura de la diáfisis femoral puede ser tratada por osteosíntesis con placa, si se respetan los principios biomecánicos que enunció Pauwels y que desarrolló posteriormente el grupo $\mathrm{AO}^{12}$. Se han descrito diferentes técnicas quirúrgicas para solucionar este problema: el cementado de los orificios de los tornillos ${ }^{11}$, la utilización de refuerzos de aloinjerto fijados con cerclajes $^{8,13-16}$, y la osteosíntesis con placas especiales, como 


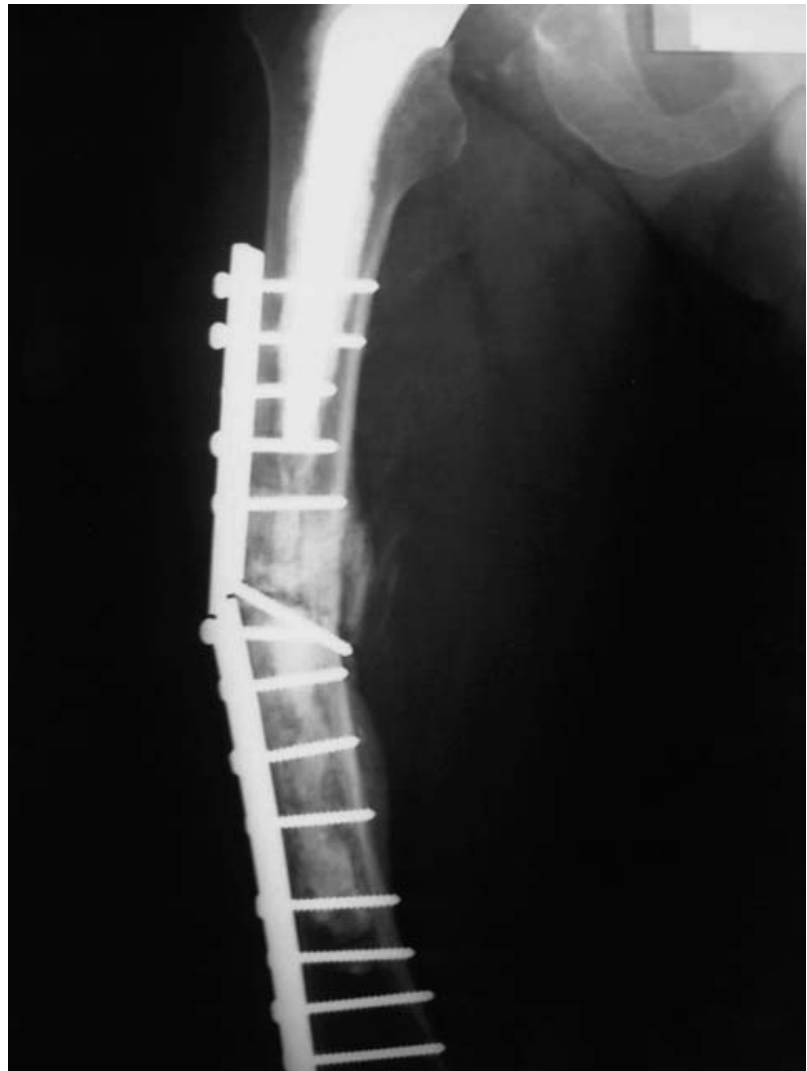

Figura 4. Mismo caso de la figura 3 (7 meses después): pseudoartrosis y rotura de la placa. Se observa cemento en el foco de la fractura.

las de Dall-Milles ${ }^{2,3,9}$ o Mennen ${ }^{4,17}$. Todas estas técnicas presentan un índice de fracasos no despreciable, sobre todo cuando se utilizan en hueso porótico. Así, por ejemplo, Noorda et $\mathrm{al}^{17}$ reúnen en un estudio retrospectivo multicéntrico 36 casos de fracturas periprotésicas inestables tratadas con la placa de Mennen. Observan un índice de complicaciones mecánicas del $31 \%$ y de pseudoartrosis del $28 \%$, y por tanto, desaconsejan el uso de este tipo de osteosíntesis. La técnica de la aplicación de una contraplaca de aloinjerto deriva, de hecho, de la técnica clásica de colocar tuercas en los tornillos cuando el anclaje en éstos era precario. En nuestra opinión las tuercas no son una buena solución porque suelen aflojarse y perder por tanto su función. Otro recurso clásico es el refuerzo del hueso fracturado mediante la aplicación de placas de aloinjerto fijadas con cerclajes s,8,12-14. $^{7}$. Nosotros pensamos que el uso de estas placas de aloinjerto como contraplaca para el anclaje de los tornillos permite una fijación mucho más firme que la que proporcionan los cerclajes. Existen estudios biomecánicos que comparan diferentes técnicas de osteosíntesis y concluyen que el montaje más estable en fracturas periprotésicas es el que utiliza una placa de osteosíntesis clásica fijada con tornillos ${ }^{18}$. En otro estudio multicéntrico (con 40 pacientes), Haddad et al desta-

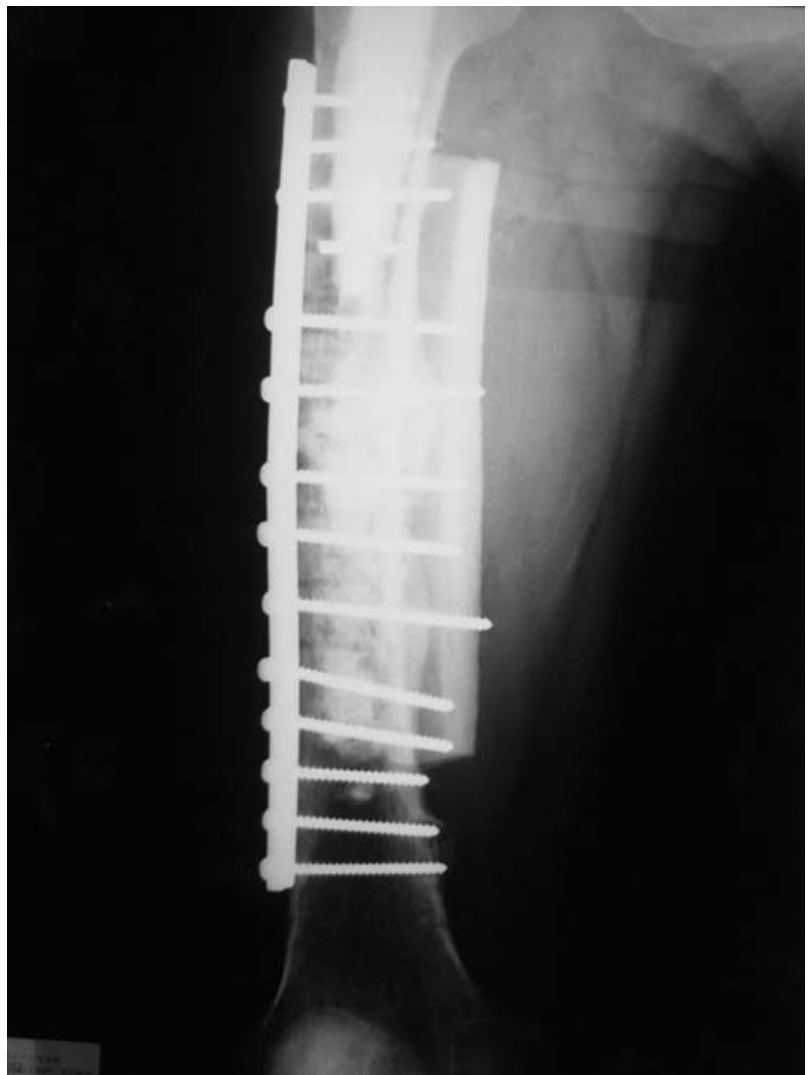

Figura 5. Mismo caso de las figuras 3 y 4. Reintervención: osteosíntesis con placa más contraplaca atornillada de aloinjerto óseo, más injerto óseo autólogo.

can la doble utilidad, mecánica y biológica, de las placas de aloinjerto óseo cortical. Al incorporarse a medio plazo (alrededor de un año), el aloinjerto queda como refuerzo permanente del hueso ${ }^{19}$.

En cuanto al cementado endomedular el manual de la Asociación de Osteosíntesis (AO), en su tercera edición, describe una técnica de cementado de los orificios de los tornillos con el objetivo de mejorar su anclaje en hueso débil $^{11}$. El cementado endomedular es pues una variante de la técnica clásica descrita por el grupo AO. En nuestra experiencia, el cementado endomedular permite un anclaje de los tornillos mucho más firme que el simple cementado de los orificios de los tornillos. No obstante, hay que ser extremadamente cuidadosos en el momento del cementado, puesto que es imprescindible evitar que el cemento penetre en el foco de fractura.

Pese a que la serie que presentamos es aún corta, las técnicas descritas se han convertido en nuestro centro en un recurso habitual para solucionar fracturas en hueso porótico. Pensamos que ambas técnicas deben ser consideradas recursos de osteosíntesis aplicables sólo en aquellos casos en los que no es posible conseguir una fijación estable con las técnicas habituales. 


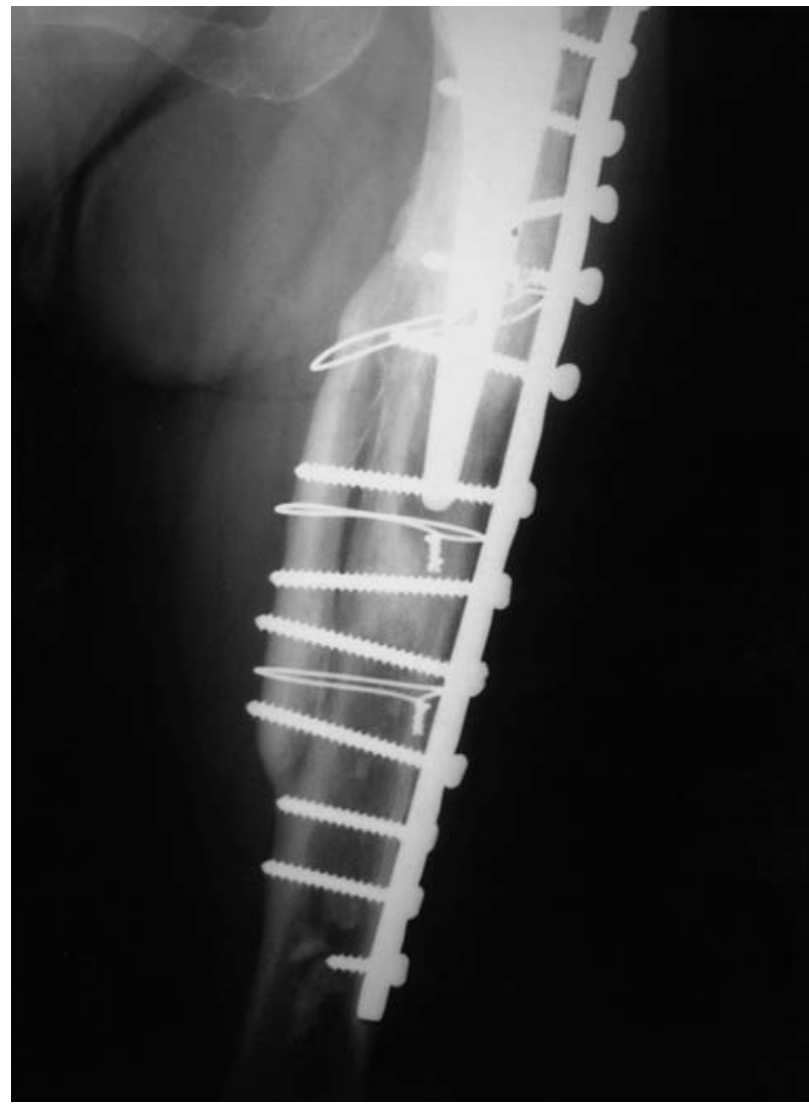

Figura 6. Contraplaca de aloinjerto congelado (tabla 3, caso n. ${ }^{\circ} 13$ ). Tras 6 años de seguimiento se logró la incorporación del aloinjerto, que quedó como refuerzo permanente del fémur.

\section{Agradecimientos}

Al Dr. Rafael Orozco Delclós por enseñarnos los fundamentos de la osteosíntesis, su ilusión permanente, y su meticulosidad en la planificación preoperatoria y en los detalles técnicos de la cirugía de las fracturas.

\section{BIBLIOGRAFÍA}

1. Campbell P, Mc Williams TG. Periprosthetic femoral fractures. Curr Orthop 2002;16:126-32.

2. Haddad FS, Marston RA, Muirhead-Allwood SK. The DallMilles cable and plate system for periprosthetic femoral fractures. Injury 1997;28:445-7.

3. Kamimeni S, Vindlacheruvu R, Ware HE. Peri-prosthetic femoral shaft fractures treated with plate and cable fixation. Injury 1999;30:261-8.

4. Kamimeni S, Ware HE. The Mennen plate: unsuitable for elderly femoral peri-prosthetic fractures. Injury 1999;30:25760.

5. Kavanagh BF. Femoral fractures associated with total hip arthroplasty. Orthop Clin North Am 1992;23:249-57.
6. Kolstad K. Revision THR after periprosthetic femoral fractures. An analysis of 23 cases. Acta Orthop Scand 1994;65: 505-8.

7. Shandu SS, Fern DE, Parsons SW. An improved cementing technique for revision hip surgery after peri-prosthetic fractures. Injury 1999;30:195-8.

8. Tower SS, Beals RK. Fractures of the femur after hip replacement. Orthop Clin North Am 1999;30:225-47.

9. Venu KM, Koka R, Garkipati R, Shenava Y, Madhu TS Dall-miles cable and plate fixation for the treatment of periprosthetic femoral fractures. Analysis of results in 13 cases. Injury 2001;32:395-400.

10. Duncan CP, Masri BA. Fractures of the femur after hip replacement. AAOS Instruct Course Lect 1995;45:293-304.

11. Müller ME, Allgöwer M, Schneider R, Willenegger H. Bases del tratamiento quirúrgico de la pseudoartrosis. En: Manual de Osteosíntesis. 3th ed. Barcelona: Springer-Verlag Ibérica, 1993; p. 720-3.

12. Orozco R. Fracturas de la diáfisis del fémur (segmento 32-) En: Orozco R, Sales JM, Videla M, editors. Atlas de osteosíntesis. Fracturas de los huesos largos. Barcelona: Masson, SA, 1998, p. 159-99.

13. Gross AE. Revision arthroplasty of the hip using allograft bone. En: Czitrom AA y Gross AE, editors. Allografts in orthopaedic practice. Baltimore: Williams \& Wilkins, 1992; p. $147-74$

14. Ramón R, Segur JM, Gallart X, García S, Riba J, Combalía A, et al. The use of proximal femoral allografts in revision total hip arthroplasty. Ann Transplantation 2001;6:30-3.

15. Rock MG. Revision Joint Replacement: proximal femoral replacement. En: Czitrom AA, Winkler, editors. Orthopaedic allograft surgery. Wien: Springer-Verlag, 1996, p. 92-100.

16. Stokey I, Gross AE. Allograft reconstruction in total knee arthroplasty. En: Czitrom AA y Gross AE, editors. Allografts in orthopaedic practice. Baltimore: Williams \& Wilkins, 1992; p. 175-96.

17. Noorda RJ, Wuisman PI. Mennen plate fixation for the tratment of periprosthetic femoral fractures: a multicenter study of thirty-six fractures. J Bone Joint Surg Am 2002;84A: 2211-5.

18. Dennis MG, Simon JA, Kummer FJ, Koval KJ, Di Cesare PE. Fixation of periprosthetic femoral shaft fractures occurring at the tip of the stem: a biomechanical study of 5 techniques. J Arthroplasty 2000;15:523-8.

19. Haddad FS, Duncan CP, Berry DJ, Lewallen DG, Gross AE, Chandler HP. Periprosthetic femoral fractures around well-fixed implants: use of cortical onlay allografts with or without a plate. J Bone Joint Surg Am 2002;84A:945-51.

Conflicto de intereses. Los autores no hemos recibido ayuda económica alguna para la realización de este trabajo. Tampoco hemos firmado ningún acuerdo por el que vayamos a recibir beneficios u honorarios por parte de alguna entidad comercial. Por otra parte, ninguna entidad comercial ha pagado ni pagará a fundaciones, instituciones educativas u otras organizaciones sin ánimo de lucro a las que estemos afiliados. 